\title{
FINITE DIFFERENCE SCHEME FOR THE LANDAU-LIFSHITZ EQUATION
}

\author{
ATSUSHI FUWA ${ }^{1}$, TETSUYA ISHIWATA ${ }^{2}$ AND MASAYOSHI TSUTSUMI ${ }^{3}$
}

\begin{abstract}
We propose a finite difference scheme for the Heisenberg equation and the LandauLifshitz equation. These equations have a length-preserving property and energy conservation or dissipation property. Our proposed scheme inherits both characteristic properties. We also show that the boundedness of finite difference solutions and an unique solvability of our scheme. Finally, we show some numerical examples.
\end{abstract}

Key words. Finite difference scheme, nonlinear partial differential equation, Landau-Lifshitz equation.

AMS subject classifications. 35K55, 35Q60, 65N06

1. Introduction. In this short report we propose a finite difference scheme for the Landau-Lifshitz equation in the form

$$
\frac{\partial u}{\partial t}=u \times \Delta u-\mu u \times(u \times \Delta u)
$$

where $u=u(x, t)=\left(u_{1}(x, t), u_{2}(x, t), u_{3}(x, t)\right): \Omega \times(0, \infty) \rightarrow \mathbb{R}^{3}, \mu$ is the Gilbert damping constant and $\times$ denotes the vector product in $\mathbb{R}^{3}$. This equation describes the evolution of spin fields in non-equilibrium continuum ferromagnets. When $\mu=0$, this equation is called Heisenberg equation. For simplicity, we consider only the case $\Omega=[-1,1]$ and boundary condition is periodic. It is obvious that the equation $(1.1)$ has a length-preserving property: $|u(x, t)|=|u(x, 0)|$ for any $t>0$. Thus, we usually assume that $|u(x, 0)|=1$ and only consider solutions in a class of vector functions which take value in a unit sphere. Note that any constant vector functions which length are one are trivial solutions of (1.1). This is also true for Neumann boundary condition case.

Let $E(u(t))$ be an energy defined by $E(u(t)):=\|\nabla u(t)\|_{L^{2}(\Omega)}^{2}$. Then the energy satisfies

$$
E(u(t))=E\left(u_{0}\right)-2 \mu \int_{0}^{t}\|u(s) \times \Delta u(s)\|_{L^{2}(\Omega)}^{2} d s
$$

for any $t>0$. That is, this problem has energy dissipation property for the case $\mu>0$ and energy conservation property for the case $\mu=0$.

The purpose of this paper is to propose a finite difference scheme which inherits the above important properties from the original problem. The contents of this paper are as follows: In Section 2, we propose a finite difference scheme and show that the scheme satisfies a length-preserving properties and inherits the property of energy. In Section 3, we show the unique solvability of the proposed scheme because the scheme

\footnotetext{
${ }^{1}$ Mizuho Information \& Research Institute, Japan.

${ }^{2}$ Department of Mathematics, Faculty of Education, Gifu university, Japan. E-mail : tisiwata@gifu-u.ac.jp

${ }^{2}$ Department of Applied Physics, School of Science and Engineering, Waseda university, Japan.
} 
is implicit and nonlinear. In Section 4, some numerical examples for exact solutions are shown to demonstrate that the proposed scheme has good features. Finally, we mention a convergence of the finite difference solution to the analytical solution. A proof of the convergence works in progress. We will report about the convergence in the forthcoming paper.

2. Proposed scheme and its properties. Let $u_{n}^{m}=\left(u_{1, n}^{m}, u_{2, n}^{m}, u_{3, n}^{m}\right)$ denote a finite difference solution at $x=x_{n}(n=0,1, \cdots, N), t=t_{m}(m=0,1,2, \cdots)$. The proposed finite difference scheme is as follows:

$$
\frac{u_{n}^{m+1}-u_{n}^{m}}{\Delta t}=u_{n}^{m+1 / 2} \times \Delta_{h} u_{n}^{m+1 / 2}-\mu u_{n}^{m+1 / 2} \times\left(u_{n}^{m+1 / 2} \times \Delta_{h} u_{n}^{m+1 / 2}\right)
$$

for $n=0,1, \cdots, N-1$ and $m=0,1,2, \cdots$,

$$
u_{0}^{m}=u_{N}^{m}, \quad u_{-1}^{m}=u_{N-1}^{m}
$$

for $m=0,1,2, \cdots$ and

$$
u_{n}^{0}=u_{0}\left(x_{n}\right)
$$

for $n=0,1,2, \cdots, N$. Here $x_{n}-x_{n-1}=\Delta x>0, t_{m}-t_{m-1}=\Delta t>0, u_{n}^{m+1 / 2}=$ $\left(u_{n}^{m+1}+u_{n}^{m}\right) / 2$ and $\Delta_{h}$ is the standard discretization of $\Delta$ :

$$
\Delta_{h} u_{n}=\frac{u_{n+1}-2 u_{n}+u_{n-1}}{\Delta x^{2}} .
$$

Hereinafter, we will show that the above scheme inherits a length-preserving property and energy structure from the original problem.

By taking an inner product of (2.1) with $u_{n}^{m+1 / 2}$, we easily obtain

$$
\left|u_{n}^{m+1}\right|=\left|u_{n}^{m}\right|
$$

for any $m$ and $n$. This means that the proposed scheme has a length-preserving property. Moreover, this fact leads to the boundedness of the finite difference solutions in $l^{\infty}$ norm.

Let $E_{h}\left(u^{m}\right)$ be the discrete energy defined by

$$
E_{h}\left(u^{m}\right):=\left\|D^{+} u^{m}\right\|_{2}^{2}
$$

Here $D^{+}$is a finite difference operator defined by

$$
D^{+} u_{n}:=\frac{u_{n+1}-u_{n}}{\Delta x}
$$

and $\|\cdot\|_{2}$ means

$$
\|v\|_{2}=\left(\sum_{n=0}^{N-1}\left|v_{n}\right|^{2} \Delta x\right)^{1 / 2} .
$$

Note that the following relationship is called "summation by parts" which is a discrete analogue of integration by parts:

$$
\sum_{n=0}^{N-1}\left(D^{-} a_{n}\right) b_{n}=-\sum_{n=0}^{N-1} a_{n}\left(D^{+} b_{n}\right)
$$


where $a_{n}$ and $b_{n}$ are periodic in $n$ and $D^{-}$is a finite difference operator defined by

$$
D^{-} u_{n}:=\frac{u_{n}-u_{n-1}}{\Delta x}
$$

By taking an inner product of (2.1) with $\Delta_{h} u_{n}^{m+1 / 2}$ and using "summation by parts" and boundary condition (2.2), we obtain

$$
E_{h}\left(u^{M}\right)=E_{h}\left(u^{0}\right)-2 \mu \sum_{m=0}^{M-1}\left\|u^{m+1 / 2} \times \Delta_{h} u^{m+1 / 2}\right\|_{2}^{2} \Delta t .
$$

The above result shows that the finite difference solution of (2.1) satisfies a discrete version of (1.2).

Remark: We here note some other methods. We first mention the projection method (for example, see [1]). This method is useful to keep the length of numerical solutions. In this procedure, we first calculate the intermediate solution $\tilde{u_{n}}$ by some scheme, for example, we may use an implicit scheme -, and next project to a unit sphere, that is, $u_{n}^{m+1}=\tilde{u_{n}} /\left|\tilde{u_{n}}\right|$. Thus, the length of numerical solutions are always equal to 1 . But there is no guarantee to inherit the energy property. Next we mention the designing methods of a finite difference scheme from viewpoint of energy structure. For example, D. Furihata proposed a procedure to design a finite difference scheme which inherits energy structure from the original problem (see [2]).

3. Unique existence of the solution to the proposed scheme. In this section we establish an unique existence of finite difference solution of (2.1) at each time step because the proposed scheme is implicit. We first show the uniqueness.

LEMmA 3.1. Suppose that there exists solution of (2.1). If

$$
\frac{\Delta t}{\Delta x^{2}}<\frac{1}{2+3 \mu}
$$

then the solution is unique.

Sketch of proof. Suppose that there are two solutions $v_{n}$ and $w_{n}$ which satisfy (2.1) as $u_{n}^{m+1}$. By using $a_{n} \times \Delta_{h} a_{n}=a_{n} \times\left(a_{n+1}+a_{n-1}\right) / \Delta x^{2}$, we have

$$
\begin{aligned}
\|w-v\|_{\infty} \leq & \frac{\Delta t}{\Delta x^{2}}\left(2\left\|\frac{w-v}{2}\right\|_{\infty} \cdot\left\|\frac{w+u^{m}}{2}\right\|_{\infty}+2\left\|\frac{v+u^{m}}{2}\right\|_{\infty} \cdot\left\|\frac{w-v}{2}\right\|_{\infty}\right. \\
& +2 \mu\left\|\frac{w-v}{2}\right\|_{\infty} \cdot\left\|\frac{w+u^{m}}{2}\right\|_{\infty}^{2}+2 \mu\left\|\frac{v+u^{m}}{2}\right\|_{\infty} \cdot\left\|\frac{w-v}{2}\right\|_{\infty} \cdot\left\|\frac{w+u^{m}}{2}\right\|_{\infty} \\
& \left.+2 \mu\left\|\frac{v+u^{m}}{2}\right\|_{\infty}^{2} \cdot\left\|\frac{w-v}{2}\right\|_{\infty}\right) \\
\leq & (2+3 \mu) \frac{\Delta t}{\Delta x^{2}}\|w-v\|_{\infty}
\end{aligned}
$$

since $\left\|\left(v+u^{m}\right) / 2\right\|_{\infty} \leq 1$ and $\left\|\left(w+u^{m}\right) / 2\right\|_{\infty} \leq 1$. Thus we have the assertion.

Next we show the existence of a solution.

THEOREM 3.2. If

$$
\frac{\Delta t}{\Delta x^{2}}<\min \left\{\frac{M-1}{M^{2}+\mu M^{3}}, \frac{1}{2 M+3 \mu M^{2}}\right\}
$$


for some $M>1$, then the solution of (2.1) exists.

We only mention the outline of proof. We use iteration:

$$
\begin{aligned}
\frac{v_{n}^{k+1}-u_{n}^{m}}{\Delta t}= & \frac{v_{n}^{k}+u_{n}^{m}}{2} \times \Delta_{h} \frac{v_{n}^{k}+u_{n}^{m}}{2} \\
& -\mu \frac{v_{n}^{k}+u_{n}^{m}}{2} \times\left(\frac{v_{n}^{k}+u_{n}^{m}}{2} \times \Delta_{h} \frac{v_{n}^{k}+u_{n}^{m}}{2}\right)
\end{aligned}
$$

and construct a sequence $\left\{v_{n}^{k}\right\}$. The next lemma shows the boundedness of $\left\{v_{n}^{k}\right\}$.

If

and

$$
\frac{\Delta t}{\Delta x^{2}} \leq \frac{M-1}{M^{2}+\mu M^{3}},
$$

then we have

$$
\left\|\frac{v^{k}+u^{m}}{2}\right\|_{\infty} \leq M
$$

for any $k \in \mathbb{N}$.

Sketch of proof. Let $w_{n}^{k}=\left(v_{n}^{k}+u_{n}^{m}\right) / 2$. Using (3.2), we obtain

$$
\begin{aligned}
w_{n}^{k+1} & =\frac{v_{n}^{k+1}-u_{n}^{m}}{2}+u_{n}^{m} \\
& =u_{n}^{m}+\frac{\Delta t}{2 \Delta x^{2}}\left(w_{n}^{k} \times\left(w_{n+1}^{k}+w_{n-1}^{k}\right)-\mu w_{n}^{k} \times\left(w_{n}^{k} \times\left(w_{n+1}^{k}+w_{n-1}^{k}\right)\right)\right) .
\end{aligned}
$$

Thus, we have

$$
\left\|w^{k+1}\right\|_{\infty} \leq 1+\frac{\Delta t}{\Delta x^{2}}\left(\left\|w^{k}\right\|_{\infty}^{2}+\mu\left\|w^{k}\right\|_{\infty}^{3}\right) .
$$

If $\left\|w^{k}\right\|_{\infty} \leq M$, then we have

$$
\begin{aligned}
\left\|w^{k+1}\right\|_{\infty} & \leq 1+\frac{\Delta t}{\Delta x^{2}}\left(M^{2}+\mu M^{3}\right) \\
& \leq M .
\end{aligned}
$$

under the conditions (3.4). Thus, we have the assertion.

Remark: If we set $v_{n}^{0}=u_{n}^{m}$, then (3.3) holds for any $M>1$.

Next we show the convergence of $\left\{v_{n}^{k}\right\}$ as $k \rightarrow \infty$. By a similar argument as in the previous two proofs, we obtain the following:

$$
\begin{aligned}
\frac{v_{n}^{k+2}-v_{n}^{k+1}}{\Delta t}= & \frac{v_{n}^{k+1}-v_{n}^{k}}{2} \times \frac{w_{n+1}^{k+1}+w_{n-1}^{k+1}}{\Delta x^{2}}+w_{n}^{k} \times \frac{v_{n+1}^{k+1}-v_{n+1}^{k}+v_{n-1}^{k+1}-v_{n-1}^{k}}{2 \Delta x^{2}} \\
& -\mu\left(\frac{v_{n}^{k+1}-v_{n}^{k}}{2} \times\left(w_{n}^{k+1} \times \frac{w_{n+1}^{k+1}+w_{n-1}^{k+1}}{\Delta x^{2}}\right)+w_{n}^{k} \times\left(\frac{v_{n}^{k+1}-v_{n}^{k}}{2} \times\right.\right. \\
& \left.\left.\frac{w_{n+1}^{k+1}+w_{n-1}^{k+1}}{\Delta x^{2}}\right)+w_{n}^{k} \times\left(w_{n}^{k} \times \frac{v_{n+1}^{k+1}-v_{n+1}^{k}+v_{n-1}^{k+1}-v_{n-1}^{k}}{2 \Delta x^{2}}\right)\right)
\end{aligned}
$$


By the previous lemma, we have

$$
\left\|v^{k+2}-v^{k+1}\right\|_{\infty} \leq\left(2 M+3 \mu M^{2}\right) \frac{\Delta t}{\Delta x^{2}}\left\|v^{k+1}-v^{k}\right\|_{\infty}
$$

and this shows that $\left\{v_{n}^{k}\right\}$ has a limit $v_{n}^{*}=\lim _{k \rightarrow \infty} v_{n}^{k}$ under the assumption (3.1). Finally, we have the theorem.

4. Numerical examples. In this section we show some numerical examples for exact solutions to verify the effectiveness of the proposed scheme.

4.1. Exact solutions. In this subsection we propose non-trivial exact solutions for Landau-Lifshitz equation (1.1) on $\Omega=[-1,1]$ with a periodic boundary condition. Let $\alpha \in \mathbb{R}$ and $l \in \mathbb{Z}$. The exact solution is given by

$$
\begin{aligned}
& u(x, t)=\left(u_{1}(x, t), u_{2}(x, t), u_{3}(x, t)\right) \\
& =\left(\frac{\sin \alpha \cos [k \cdot x-\phi(x, t ; \alpha, k, \mu)]}{d(t ; \alpha, k, \mu)}, \frac{\sin \alpha \sin [k \cdot x-\phi(x, t ; \alpha, k, \mu)]}{d(t ; \alpha, k, \mu)}, \frac{e^{k^{2} \mu t} \cos \alpha}{d(t ; \alpha, k, \mu)}\right)
\end{aligned}
$$

where $k=l \pi, d(t ; \alpha, k, \mu)=\sqrt{\sin ^{2} \alpha+e^{2 k^{2} \mu t} \cos ^{2} \alpha}$ and

$$
\phi(x, t ; \alpha, k, \mu)=\frac{1}{\mu} \log \left(\frac{d(t ; \alpha, k, \mu)+e^{k^{2} \mu t} \cos \alpha}{1+\cos \alpha}\right) .
$$

Note that we can easily obtain the exact solution for higher dimensional case.

Letting $\mu \rightarrow 0+$, we have

$$
\begin{aligned}
& u_{1}(x, t)=\sin \alpha \cos \left(k \cdot x-\left(|k|^{2} \cos \alpha\right) t\right), \\
& u_{2}(x, t)=\sin \alpha \sin \left(k \cdot x-\left(|k|^{2} \cos \alpha\right) t\right), \\
& u_{3}(x, t)=\cos \alpha .
\end{aligned}
$$

We see that the function $u(x, t)=\left(u_{1}(x, t), u_{2}(x, t), u_{3}(x, t)\right)$ is an exact solution for Heisenberg equation. This solution is already shown in [4].

Remark: In [3], Lakshmanan and Nakamura propose a method for constructing the exact solution of Landau-Lifshitz equation. However, their proposed procedure is not valid in general (see [5]). Thus, we cannot obtain the above exact solution by their method.

4.2. Numerical results. Figure 4.1 and 4.2 show numerical results for exact solutions by the proposed scheme with $\Delta x=1 / 30, \Delta t=1 / 20000$.

Figure 4.1 shows a view of $u_{1}$ of the numerical solution in (a), a view of $u_{1}$ of the exact solution given in the previous subsection in (b) and a behavior of energy in (c) in the case $\mu=0$. We can see that the conservation of energy holds.

Figure 4.2 shows a view of $u_{1}$ of the numerical solution in (a), a view of $u_{1}$ of the exact solution given in the previous subsection in (b) and a behavior of energy in (c) in the case $\mu=0.1$. We can see that the dissipation of energy holds and the behavior of energy of the numerical solution is a very good agreement with that of the exact solution.

From the numerical experiments, we may conclude that the proposed scheme has good features. 


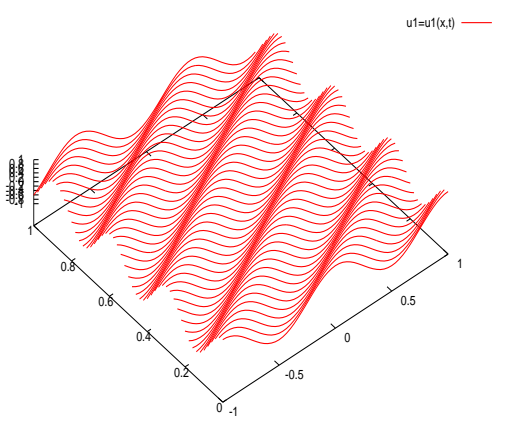

(a) $u_{1}$ of the numerical solution

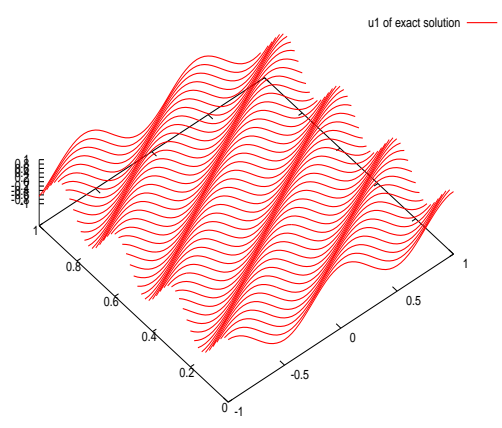

(b) $u_{1}$ of the exact solution

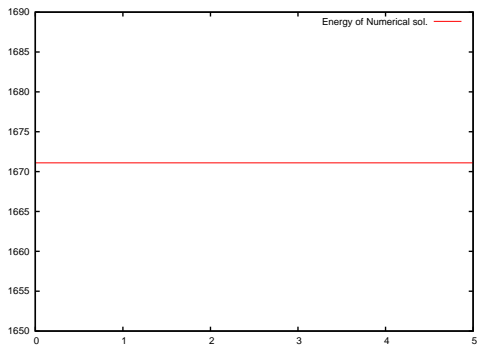

(c) behavior of energy of the numerical solution

FIG. 4.1. Numerical results for $\mu=0$

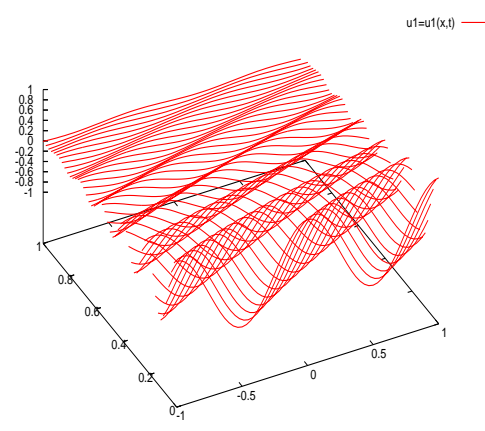

(a) $u_{1}$ of the numerical solution

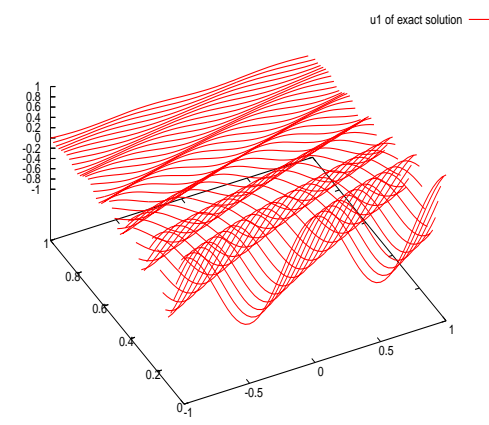

(b) $u_{1}$ of the exact solution

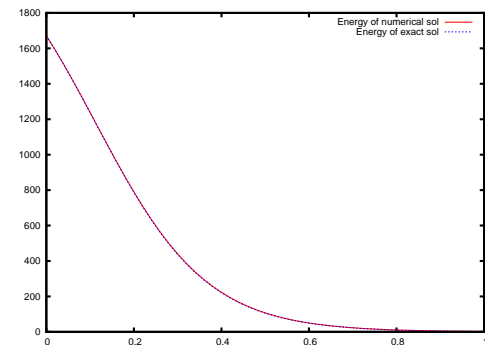

(c) behavior of the energy of numerical solution (solid line) and the exact solutions (dotted line)

FIG. 4.2. Numerical results for $\mu=0.1$ 
Acknowledgment. We would like to thank the referee for her or his comments and suggestions. The second author is partially supported by Grant-in-Aid for Encouragement of Young Scientists (No. 18740048).

\section{REFERENCES}

[1] E. Weinan, And XiaO-Ping Wang, Numerical Methods for the Landau-Lifshitz Equation, SIAM J. Numer. Anal. 38, No. 5 (2000), 1647-1665.

[2] D. Furihata, Finite Difference Schemes for $\frac{\partial u}{\partial t}=\left(\frac{\partial}{\partial x}\right)^{\alpha} \frac{\delta G}{\delta u}$ That Inherit Energy Conservation or Dissipation Property, J. Comput. Phys. 156 (1999), 181-205.

[3] M. LaKshmanan, AND K. NAKAmura, Landau-Lifshitz Equation of Ferromagnetism: Exact Treatment of the Gilbert Damping, Physical Review Letters 53 (1984), 2497.

[4] M. Lakshmanan, Th.W. Rijgrok, and C.J. Thompson, On the Dynamicals of a Continuum Spin System, Physca 84A (1976), 577-590.

[5] E. Magyari, H. Thomas, and R. Weber, Comment on "Landau-Lifshitz Equation of Ferromagnetism: Exact Treatment of the Gilbert Damping", Physical Review Letters 56 (1986), 1756. 